\title{
Preface to the Special Issue on Graph Data Management in Online Social Networks
}

\author{
Kai Zheng ${ }^{1} \cdot$ Guanfeng $\mathrm{Liu}^{2} \cdot$ Mehmet A. Orgun ${ }^{2} \cdot$ Junping $\mathrm{Du}^{3}$ \\ Published online: 16 January 2020 \\ C) Springer Science+Business Media, LLC, part of Springer Nature 2020
}

We are delighted to present this special issue of World Wide Web on Graph Data Management in Online Social Networks. Recent years have witnessed the explosion of graph data generated from a wide range of enterprises and applications at an unprecedented way. Graph data management, which concerns techniques in modelling, storing, querying, and learning graph data has been found particular useful in online social network (OSN) analysis, such as expert finding, social community mining and social position detection. The purpose of this special issue is to bring together some recent and significant results in analysing OSNs with novel graph data management techniques in a timely fashion.

The guest editors selected 11 contributions that covers varying topics within this theme, ranging from graph-based representations to learning models, from graph-based query processing to analysis. Many problems in this area share common methods including graph data structures and deep learning models.

Li et al. in "Semi-supervised Clustering with Deep Metric Learning and Graph Embedding" propose a novel semi-supervised clustering approach based on deep metric learning and graph embedding, which enhances the robustness of metric learning network and promotes the accuracy of clustering.

This article belongs to the Topical Collection: Special Issue on Graph Data Management in Online Social Networks

Guest Editors: Kai Zheng, Guanfeng Liu, Mehmet A. Orgun, and Junping Du

Kai Zheng

zhengkai@uestc.edu.cn

Guanfeng Liu

guanfeng.liu@mq.edu.au

Mehmet A. Orgun

mehmet@ics.mq.edu.au

Junping Du

junpingdu@126.com

1 University of Electronic Science and Technology of China, Chengdu, Sichuan, China

2 Macquarie University, NSW, Macquarie Park 2109, Australia

3 Beijing University of Posts and Telecommunications, Haidian, Beijing 100876, China 
In "Gaussian Mixture Embedding of Multiple Node Roles in Networks", the authors propose to embed a single node as a mixture of Gaussian distribution in a low-dimensional space. Each Gaussian component corresponds to a latent role that the node plays. The proposed approach thus can characterize network nodes in a comprehensive representation, especially bridging nodes, which are relevant to different communities.

Yu et al. in "A Relationship Extraction Method for Domain Knowledge Graph Construction" obtain upper and lower relationships from structured data in the classification system of network encyclopedia and semi-structured data in the classification labels of web pages, and extract non-superordinate relationships from unstructured text through the proposed convolution residual network based on improved cross-entropy loss function.

In "Cluster Query: a New Query Pattern on Temporal Knowledge Graph", the authors introduce a novel cluster query to retrieve forward-backward correlation facts from temporal knowledge graph and study five corresponding basic query patters of unit matching and complete matchings.

In "Towards k-Vertex Connected Component Discovery from Large Networks", the authors study a k-vertex connected component ( $\mathrm{k}-\mathrm{VCC})$ model, which is much more cohesive, and thus supports overlapping between components well.

Yin et al. in "Matching of Social Events and Users: A Two-Way Selection Perspective" propose a novel two-stage framework for social event participation analysis by adapting the classic Gale-Shapley algorithm for stable matching.

Fang et al. in "Distributed and Parallel Processing for Real-time and Dynamic Spatio-temporal Graph" propose a computational model suitable for handling the spatio-temporal graph in Distributed Stream Processing Engine (DSPE), by reconstructing the DSPE's parallel processing slots.

In "Dolha - an Efficient and Exact Data Structure for Streaming Graphs" the authors present a new memory efficient graph data structure called Dolha to support massive graph storage and frequent update in modern applications.

Qian et al. in "Detecting Topic-level Influencers in Large-Scale Scientific Networks" propose a sparse link topic model (SLTM) to detect topic-level influencers from a large collection of links between nodes and textual contents in scientific networks.

$\mathrm{Li}$ et al. in "Misinformation-oriented Expert Finding in Social Networks" focus on how to obtain an appropriate matching between the suspect misinformation and corresponding experts, and propose a multi-topic expert finding method, called LTM (List based Topic Model), to sufficiently utilize crowdsourcing wisdom.

In "Detecting the evolving community structure in dynamic social networks" the authors introduce a migration operator cooperating with efficient operators to confirm that nodes and their most neighbors are grouped together, and use a genome matrix which codes the structure information of networks to expand the search space.

The guest editors believe the papers appearing in this issue represent the frontiers of current topics in the field of graph data management and hope these articles will stimulate further development in this area. The editors express their appreciation to the authors and reviewers for contributing to this special issue.

We hope you enjoy this special issue and take some inspiration from it for your own future research.

Kai Zheng, Guanfeng Liu, Mehmet A. Orgun and Junping Du

$10 / 12 / 2019$

Publisher's note Springer Nature remains neutral with regard to jurisdictional claims in published maps and institutional affiliations. 